\title{
Cell-Matrix Interactions Modulate Interstitial Collagenase Expression by Human Keratinocytes Actively Involved in Wound Healing
}

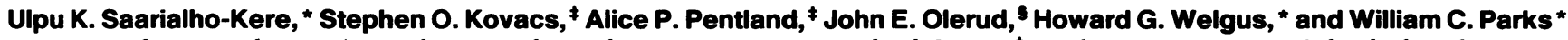
Divisions of Dermatology at * Jewish Hospital, Washington University Medical Center, ${ }^{\ddagger}$ Washington University School of Medicine, St. Louis, Missouri 63110; and the ${ }^{\S}$ University of Washington, Seattle, Washington 98195

\begin{abstract}
We reported that interstitial collagenase is produced by keratinocytes at the edge of ulcers in pyogenic granuloma, and in this report, we assessed if production of this metalloproteinase is a common feature of the epidermal response in a variety of wounds. In all samples of chronic ulcers, regardless of etiology, and in incision wounds, collagenase mRNA, localized by in situ hybridization, was prominently expressed by basal keratinocytes bordering the sites of active re-epithelialization indicating that collagenolytic activity is a characteristic response of the epidermis to wounding. No expression of mRNAs for 72- and 92-kD gelatinases or matrilysin was seen in keratinocytes, and no signal for any metalloproteinase was detected in normal epidermis. Immunostaining for type IV collagen showed that collagenase-positive keratinocytes were not in contact with an intact basement membrane and, unlike normal keratinocytes, expressed $\alpha_{5} \beta_{1}$ receptors. These observations suggest that cellmatrix interactions influence collagenase expression by epidermal cells. Indeed, as determined by ELISA, primary cultures of human keratinocytes grown on basement membrane proteins (Matrigel; Collaborative Research Inc., Bedford, MA) did not express significant levels of collagenase, whereas cells grown on type I collagen produced markedly increased levels. These results suggest that migrating keratinocytes actively involved in re-epithelialization acquire a collagenolytic phenotype upon contact with the dermal matrix. (J. Clin. Invest. 1993. 92:2858-2866.) Key words: collagenase $\bullet$ keratinocytes $\bullet$ wound healing • basement membrane • collagen
\end{abstract}

\section{Introduction}

Wound healing is an orderly process that involves inflammation, re-epithelialization, matrix deposition, and tissue remodeling. In most injuries, especially chronic wounds, healing is accompanied by inflammation, angiogenesis, and formation of granulation tissue. Degradation of extracellular matrix is required to remove damaged tissue and provisional matrices and to permit vessel formation and cell migration, and these remodeling processes involve various proteinases. The matrix metalloproteinase gene family includes interstitial collagenase, which has the unique ability to cleave fibrillar types I, II, III, and $X$ collagens $(1,2)$, and stromelysins- 1 and -2 and matrily-

Address correspondence to Dr. William C. Parks, Dermatology Division, Jewish Hospital, 216 South Kingshighway Boulevard, St. Louis, MO 63110. 1993.

Received for publication 7 May 1993 and in revised form 22 July

J. Clin. Invest.

(c) The American Society for Clinical Investigation, Inc.

$0021-9738 / 93 / 12 / 2858 / 09 \$ 2.00$

Volume 92, December 1993, 2858-2866 sin, which have a broad substrate specificity and can cleave fibronectin, laminin, pepsin-sensitive collagen regions, and proteoglycans (3). The 72- and $92-\mathrm{kD}$ gelatinases cleave native types IV, V, VII, and X collagens, and gelatins of all collagen types $(4,5)$, and, like matrilysin, are also elastolytic (5). As demonstrated in numerous cell culture studies, the pattern of active expression of metalloproteinases is cell-type specific, and is regulated by inflammatory agents such as cytokines and bacterial endotoxin (6). Regulation of enzyme activity also occurs extracellularly by controlled activation of the proenzyme forms and by inhibition of activated species by the specific tissue inhibitors of metalloproteinases (TIMPs ${ }^{1}$; reference 6).

Many in vitro studies have suggested that collagenase is primarily produced by fibroblasts and macrophages, but in our in vivo work, we reported that in ulcerated pyogenic granulomas basal keratinocytes prominently express interstitial collagenase at the advancing edges of the ulcer (7). These observations implicated the keratinocyte as a major participant in the degradation of extracellular matrix during wound healing. The results we now present here demonstrate that collagenase expression by migrating keratinocytes is an invariable feature of a disrupted epidermis, both as a consequence of normal wound healing by secondary intention and in ulceration resulting from a variety of disease processes.

Basal keratinocytes normally rest on basement membrane composed of laminin, entactin, proteoglycans, and type IV collagen (8) and other molecules, such as kalinin and other laminin variants (9). During wound healing, keratinocytes from the edge of the wound migrate over a provisional matrix of fibrin and fibronectin (10), whereas laminin inhibits keratinocyte migration (11). The interactions of cells with components of the extracellular matrix, mediated chiefly by the integrin family of heterodimeric transmembrane adhesion receptors (12), play an important role in tissue repair and regeneration. Since we did not detect expression of interstitial collagenase in our previous studies of the epidermis of nonulcerated pyogenic granulomas (7), we reasoned that exposure of keratinocytes to the underlying dermal stroma may be necessary for inducing collagenolytic activity. To elucidate the spatial interactions of cell-matrix interrelationships and keratinocyte collagenase production, we examined the distribution of $\alpha_{5} \beta_{1}$ integrin, the principal fibronectin receptor, and type IV collagen, as a marker of basement membrane integrity. We report here that collagenase and $\alpha_{5} \beta_{1}$ integrin are co-expressed by basal keratinocytes migrating over areas devoid of basement membrane. These in vivo observations indicate that the phenotype of keratinocytes is altered as they contact the dermal matrix, a finding that was reproduced in vitro by epidermal cells grown on type I collagen.

1. Abbreviation used in this paper: TIMPs, tissue inhibitors of metalloproteinases. 


\section{Methods}

Tissues. Formalin-fixed, paraffin embedded histological specimens were obtained from the Department of Pathology, Washington University School of Medicine. 17 ulcers and 7 ulcerated pyogenic granulomas from different parts of the body were studied. Several ulcers were nonspecific and were characterized by inflammation and granulation tissue. 5 specimens were stasis ulcers, 5 were decubitus ulcers, 1 was pyoderma gangrenosum, and 1 specimen exhibited marked fibrosis. In addition, 4 specimens of bullous pemphigoid, 3 specimens of pemphigus vulgaris, and 4 samples of normal skin from different parts of the body were also studied.

For acute wounds, partial thickness wounds were created under aseptic conditions with Simplate-II bleeding-time devices (OrganonTeknika, Durham, NC) on the lateral leg and/or ventral forearm of four normal elderly subjects ranging in age from 61 to $72 \mathrm{yr}$ as described (13). At 1, 3, 4, and $7 \mathrm{~d}$ after wounding, 4-mm punch biopsies were taken at the Division of Dermatology, University of Washington, from two independent wounds for each time point. The wounds were bisected in a plane perpendicular to the long axis of the wound, fixed in $0.1 \mathrm{M}$ paraformaldehyde, and embedded in paraffin.

In situ hybridization. In situ hybridization was performed as described (14). All sections $(5 \mu \mathrm{m})$ were treated with proteinase $\mathrm{K}$ (Sigma Chemical Co., St. Louis, MO) and washed in $0.1 \mathrm{M}$ triethanolamine buffer containing $0.25 \%$ acetic anhydride. Sections were covered with 25 to $50 \mu$ l of hybridization buffer containing $2.5-4 \times 10^{4} \mathrm{cpm} / \mu \mathrm{l}$ of ${ }^{35} \mathrm{~S}$-labeled RNA probe. Specimens were incubated at $55^{\circ} \mathrm{C}$ for $18 \mathrm{~h}$ in a humidified chamber. After hybridization, slides were washed under stringent conditions and processed for autoradiography as described (7). After a 3-24 d autoradiographic exposure, the photographic emulsion was developed, and the slides were stained with hematoxylineosin.

RNA probes. Probes for interstitial collagenase $(14,15), 92-\mathrm{kD}$ gelatinase (16), and TIMP-1 (17) have been described. A 1,212-bp AccI-SmaI fragment of the 3' end of 72-kD gelatinase cDNA (18) was subcloned into Bluescript KS transcription vector (Stratagene Corp., La Jolla, CA ). An 800-bp Xbal-XmnI fragment of the 5' end of matrilysin cDNA was subcloned into pGEM-7Zf $(+)(19)$ and was generously provided by Dr. Lynn M. Matrisian, Vanderbilt University, Nashville, TN. As a control for nonspecific hybridization, sections in each experiment were hybridized with ${ }^{35}$ S-labeled sense RNA transcribed from a bovine tropoelastin cDNA. The validity of this probe as a negative control has been confirmed by Northern (14) and by in situ hybridization assays $(7,20)$. In vitro transcribed RNA was labeled with $\alpha\left[{ }^{35} \mathrm{~S}\right] \mathrm{UTP}(>1,200 \mathrm{Ci} / \mathrm{mmol})$ using reagents from Promega Corp. (Madison, WI) by a modification of our previously described method (14). To increase the specific activity of the radiolabeled probes, 150 $\mu \mathrm{Ci}$ of $\alpha\left[{ }^{35} \mathrm{~S}\right] \mathrm{UTP}$ was dried in a microfuge tube under vacuum with gentle centrifugation. This effectively increases the concentration of radiolabeled precursor greater than fivefold compared to our previous protocol. Transcription reagents, $5 \mathrm{U}$ of the appropriate RNA polymerase, and $1 \mu \mathrm{g}$ of linearized DNA template were added in a total volume of $20 \mu \mathrm{l}$, and the mixture was incubated for $1 \mathrm{~h}$ at $37^{\circ} \mathrm{C}$. At this point, an additional $5 \mathrm{U}$ of polymerase were added, and the reaction was continued for $3 \mathrm{~h}$ more. These modifications have generally resulted in a 5- to 10-fold increase in the specific activity of the RNA probes. The specificity of the interstitial collagenase, 92- and 72-kD gelatinases, matrilysin, and TIMP-1 probes for the appropriate sized mRNA has been verified by blot hybridization $(19,21,22)$.

Antibodies. Polyclonal rabbit antiserum raised against a monospecific synthetic peptide from the $\alpha_{5}$ subunit of the $\alpha_{5} \beta_{1}$ integrin fibronectin receptor and antihuman $\alpha 1$ (IV) collagen monoclonal antibodies were supplied by Dr. John A. McDonald, Mayo Clinic-Scottsdale, AZ. The specificity of the $\alpha_{5}$ subunit antibody has been characterized (23). The mouse type IV collagen antibody was raised against the $\mathrm{NH}_{2}$-terminal domain of the molecule and is specific as determined by ELISA and immunoblotting (Crouch, E. C., personal communication). Polyclonal rabbit anti-human collagenase antiserum was affinity purified as described $(24,25)$. Highly purified human collagenase protein isolated from fibroblast conditioned medium (26) was coupled to CNBR Sepharose 4B (Pharmacia LKB, Uppsala, Sweden), and specific antibodies were adsorbed and eluted as described (25). As determined by ELISA, full immunoreactivity was recovered by elution with $0.1 \mathrm{M}$ glycine, $\mathrm{pH}$ 2.5. Polyclonal antiserum to laminin was purchased from Sigma Chemical Co. (LAM-89).

Immunohistochemistry. On sections serial to those used for in situ hybridization, immunostaining for $\alpha_{5} \beta_{1}$ integrin, collagenase, type IV collagen, and laminin was done by the peroxidase-antiperoxidase technique (Vectastain ABC Kit; Vector Labs, Burlingame, CA) using diaminobenzidine as a chromogenic substrate. Endogenous peroxidase was blocked by incubation with $0.3 \% \mathrm{H}_{2} \mathrm{O}_{2}$ for $30 \mathrm{~min}$ at room temperature and $10 \mathrm{mg} / \mathrm{ml}$ trypsin was applied on the slides for $30 \mathrm{~min}$. The $\alpha_{5} \beta_{1}$, laminin, and type IV collagen antibodies were diluted 1:500, $1: 2,000$, and 1:1,000, respectively, and were incubated on sections for 1 $h$ at room temperature. The collagenase antibody was diluted 1:2,000 and reacted at $4^{\circ} \mathrm{C}$ overnight. Sections were counterstained with Harris hematoxylin. Controls were performed with the appropriate rabbit preimmune serum or preimmune mouse ascites fluid.

Keratinocyte culture. Human keratinocytes were obtained from reduction mammoplasties or abdominoplasties. The subcutaneous fat and deep dermis were removed, the skin was cut into thin pieces, and the tissue was incubated in $0.25 \%$ trypsin in PBS (27). After $16 \mathrm{~h}$, the epidermis was separated from the dermis, and the keratinocytes were scraped into DME. The keratinocyte solution was added to fresh DME supplemented with $5 \%$ FCSr, $1 \%$ Hepes, and penicillin/streptomycin. Under these conditions, the keratinocytes differentiate and stratify similar to cells in vivo. Cultures were plated in duplicate 12-well plastic plates precoated with either type I collagen (Vitrogen 100; Celtrix Laboratories, Palo Alto, CA) in concentrations from 12 to $750 \mu \mathrm{g} / \mathrm{ml}$, or a basement membrane-like substratum (Matrigel; Collaborative Research Inc., Bedford, MA) in concentrations ranging from 47 to 3,000 $\mu \mathrm{g} / \mathrm{ml}$. After reaching confluence, the cells were washed, then refed. The medium was collected $72 \mathrm{~h}$ later, and the levels of accumulated interstitial collagenase were measured by a competitive ELISA as described (28). This assay has nanogram sensitivity, is specific for collagenase, and measures total enzyme present whether free or bound to TIMP or substrate, or whether in an inactive or active form.

\section{Results}

To assess if expression of collagenase is a common feature of the epidermal response to wounding, 24 samples of ulcerated lesions representing various underlying pathologies (see Methods) were studied using in situ hybridization. In all 24 samples, prominent signal for interstitial collagenase mRNA was detected in basal keratinocytes at the edges of the wounds (Figs. 1 $A$ and $B$ and $2 A$ and $B$ ). Immunostaining with affinity purified collagenase antibodies verified that basal keratinocytes produced collagenase protein with the strongest cellular staining at the wound edge (Fig. $1 F$ ). Strong immunostaining for collagenase protein was also seen in the extracellular space near to the wound indicating the presence of secreted enzyme (Fig. $1 F$ ). In a few samples $(3 / 24)$, collagenase mRNA was also present in keratinocytes some distance from the ulcer in areas of papillomatotic and hyperplastic epidermis (data not shown). In addition, collagenase expression was also seen in most samples $(22 / 24)$ in occasional fibroblast-like or macrophage-like cells in the dermis near to the ulceration (Fig. $1 A, B$, and $E$ ). Typically, however, the signal in these stromal cells was much less than that seen in the basal keratinocytes. In one ulcer characterized by fibrosis with no inflammation, collagenase mRNA was detected exclusively at the epidermal wound edge. As we reported before $(7,20)$, no signal for collagenase mRNA was 

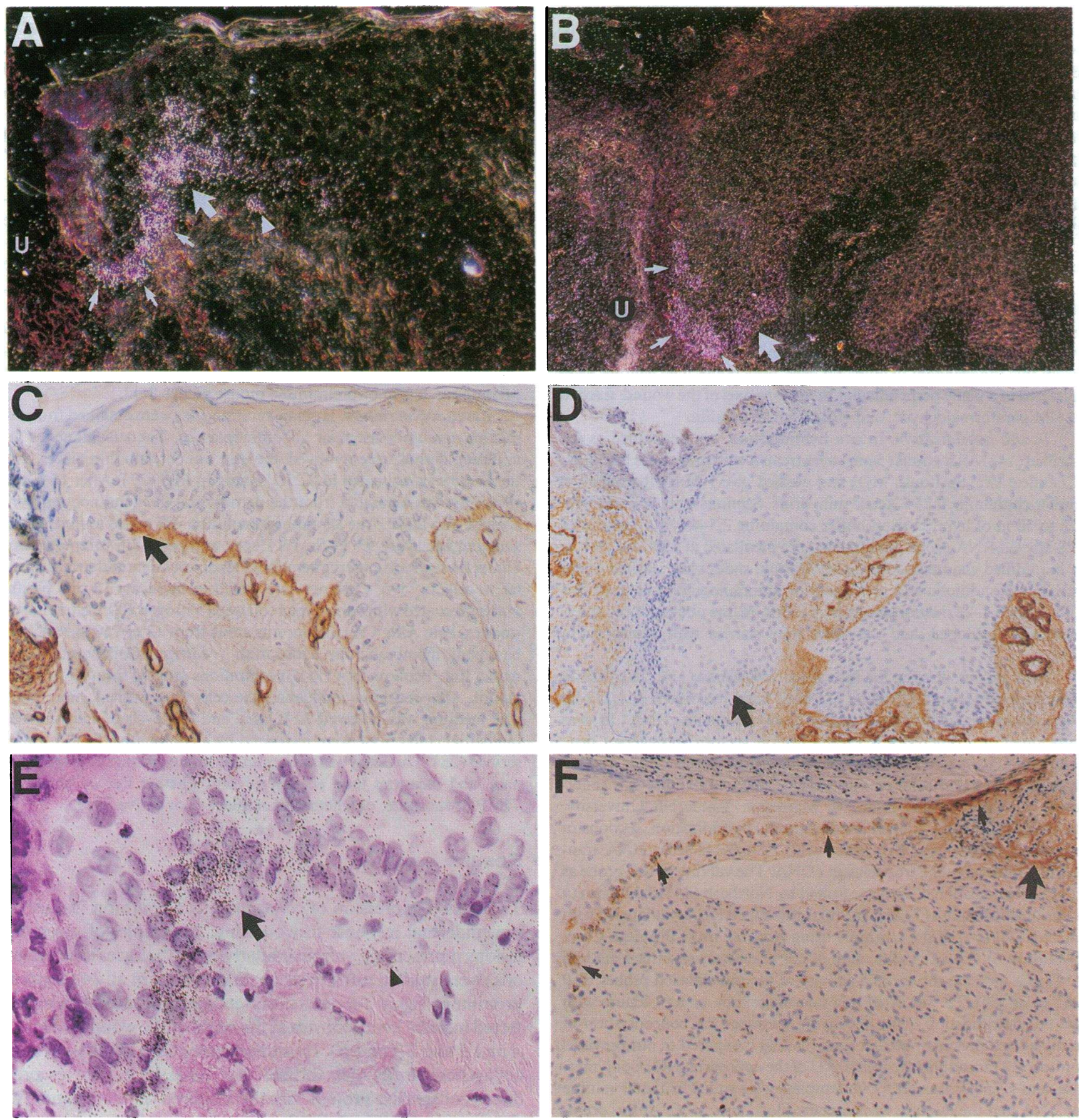

Figure 1. Collagenase mRNA is expressed by basal keratinocytes which are not in contact with a basement membrane. Serial sections were hybridized with ${ }^{35}$ S-labeled antisense RNA for collagenase mRNA $(A, B, E)$ or stained with a monoclonal antibody to type IV collagen $(C, D)$. Low power dark-field photomicrographs from ulcers of the forehead $(A)$ and buttock $(B)$ revealed strong autoradiographic signal for collagenase mRNA (small arrows) in migrating keratinocytes at the edge of the ulcer $(U)$, which is at the far left in each micrograph. In many samples, occasional stromal cells had signal for collagenase mRNA (arrowhead in $A$ and $E$, and cells in the inflamed area just to the left of the migrating epidermis in $B$ ). The large arrow approximates the extent of the basement membrane. Autoradiographic exposure was for $10 \mathrm{~d}$, and sections were stained with hematoxylin and eosin. $(E)$ High power bright-field view $(\times 210)$ of the section shown in $A$ reveals that the collagenase-positive cells are confined to the basal epidermis, and again the large arrow approximates the extent of the basement membrane. ( $C$ and $D)$ Sections parallel to $A$ and $B$ were immunostained for type IV collagen, which demonstrates that the epidermal basement membrane ceases (arrow) about where collagenase expression begins. Strong immuriostaining is evident in the basement membrane of multiple blood vessels. $(A$ and $C) \times 100$; $(B$ and $D) \times 50$. $(F)$ Immunostaining with affinity purified collagenase antibodies demonstrates the presence of enzyme protein in basal keratinocytes (small arrows). In this specimen, the ulceration is on the right side of the micrograph. Stromal staining for collagenase protein is indicated (large arrow). $\times 50$. Immunohistochemical slides were stained with Harris hematoxylin. 
seen in lymphocytes, polymorphonuclear leukocytes, in areas of unaffected epidermis, or in normal skin specimens. No specific signal was detected in samples hybridized with ${ }^{35} \mathrm{~S}$-labeled sense RNA probe (data not shown).

To characterize the tissue microenvironment of the collagenase-positive keratinocytes, we stained serial sections with antibodies for type IV collagen as a marker of the basement membrane. In all samples, collagenase-positive keratinocytes were not in contact with an intact basement membrane, whereas epidermal cells behind the migrating front, which did not express collagenase mRNA, resided on an intact basement membrane (Fig. $1 A$ and $C, B$ and $D$ ). In many samples, a few basal keratinocytes just within the area of intact basement membrane were positive for collagenase mRNA, but this signal decreased progressively in cells away from the migrating front of epidermis. The integrity of the epidermal basement membrane was further confirmed by laminin immunostaining, which coincided with that for type IV collagen (data not shown).
These observations indicate that basal keratinocytes at the edge of wounds are in contact with and migrate over dermal matrix components. As shown by others $(12,29)$, the expression of matrix receptors is altered in activated basal keratinocytes. In particular, expression of the fibronectin integrin receptor, $\alpha_{5} \beta_{1}$, is induced in migrating keratinocytes. In chronic wounds, we found that the $\alpha_{5}$ subunit of this receptor was expressed on the basal surfaces and occasionally on the pericellular edges of the same population of migrating keratinocytes that were positive for collagenase mRNA expression (Fig. 2). As expected, strong staining for $\alpha_{5} \beta_{1}$ was present in blood vessel walls of all samples. In agreement with others $(12,29)$, no staining for $\alpha_{5} \beta_{1}$ was noted in the intact epidermis or in normal skin.

Our findings demonstrate that collagenase expression is a predictable feature of keratinocytes at the edge of chronic ulcers. To assess if enzyme expression is also induced during the healing of acute normal wounds, we examined samples of
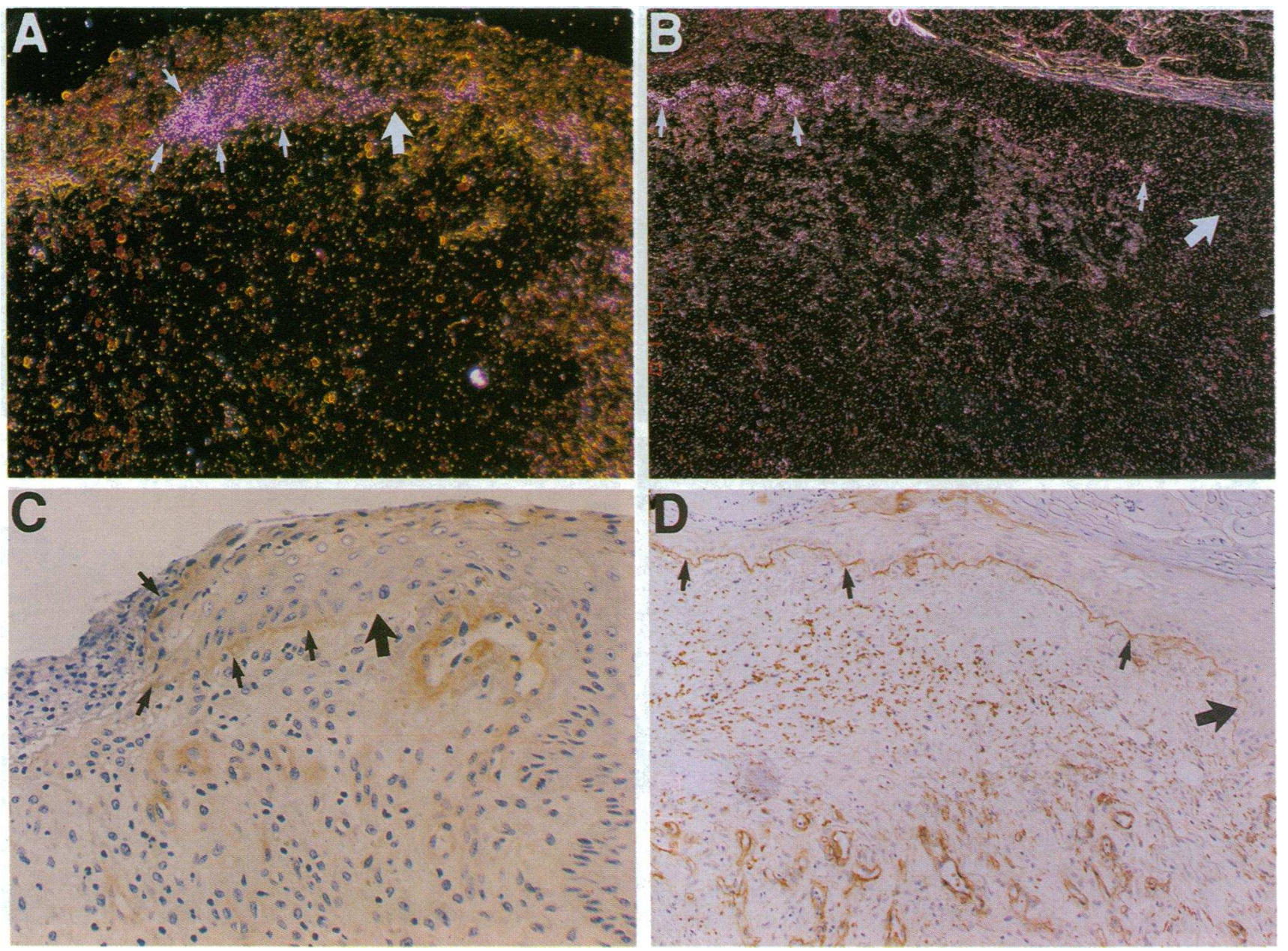

Figure 2. $\alpha_{5} \beta_{1}$ integrin is expressed by collagenase-positive keratinocytes. Signal for collagenase mRNA in a fibrotic decubitus ulcer $(A)$ and a stasis ulcer of the ankle $(B)$ was seen in migrating keratinocytes (small arrows) at the edge of the ulcer, which is at the far left in each micrograph. Large arrows approximate the extent of signal for $\alpha_{5}$ immunostaining. Autoradiography was for $10 \mathrm{~d}$. ( $C$ and $D$ ) Sections parallel to $A$ and $B$ were stained for the $\alpha 5$ subunit of the $\alpha_{5} \beta_{1}$ fibronectin receptor. Small arrows indicate staining for $\alpha_{5}$ along the basal layer of keratinocytes. In $C$, receptor staining was confined to the same population of basal keratinocytes, which express collagenase mRNA as seen in $A$. In $D$, receptor staining was strongest in areas where collagenase mRNA was expressed, as seen in $B$, then diminished about where the signal for collagenase mRNA ceased (large arrow). Strong immunostaining for $\alpha_{5}$ was predictably evident around blood vessels. Magnification: $(A$ and $C) \times 150 ;(B$ and $D) \times 60$. 
sterile partial thickness wounds made on healthy volunteers. As for the chronic ulcer samples, collagenase mRNA was expressed in basal keratinocytes at the healing edges of the normal wounds at $2 \mathrm{~d}$ after injury (Fig. $3 A$ and $C$ ), although the signal was noticeably weaker than that in the chronic wounds. By $4 \mathrm{~d}$, re-epithelialization was complete, and collagenase expression was no longer detected (Fig. $3 B$ and $D$ ). In agreement with our findings on chronic wounds, immunostaining for type IV collagen showed that the collagenase positive cells were not in contact with a basement membrane, whereas by $4 \mathrm{~d}$, the basement membrane had reassembled and no signal for enzyme mRNA was seen (Fig. 3). No signal for collagenase mRNA was seen in any dermal cell in these noninflamed acute samples.

Because the complete breakdown of cleaved fibrillar collagen may involve gelatinases, we examined the expression of other metalloproteinases in the chronic ulcer samples. No signal for the 72- and 92-kD gelatinase mRNAs was seen in the epidermis of any ulcerative or normal sample examined, even after an extended autoradiographic exposure of $17 \mathrm{~d}$. However, if eosinophils were present (in 6 of 13 samples examined), a positive signal for 72- and 92-kD gelatinase mRNAs was detected in these granulocytes (data not shown) in agreement with our previous findings $(20,21,25)$. The presence of $92-\mathrm{kD}$ protein was also examined in five samples by using an affinity purified antibody (25) and no immunoreactivity was ever localized to the epidermal ulcer edges (not shown). As expected, however, neutrophils, which contain but do not actively synthesize the enzyme (25), and eosinophils stained positively for 92-kD gelatinase protein. Since matrilysin may activate procollagenase (30) we examined if it was expressed in wounds, but no signal was detected by either immunostaining or in situ
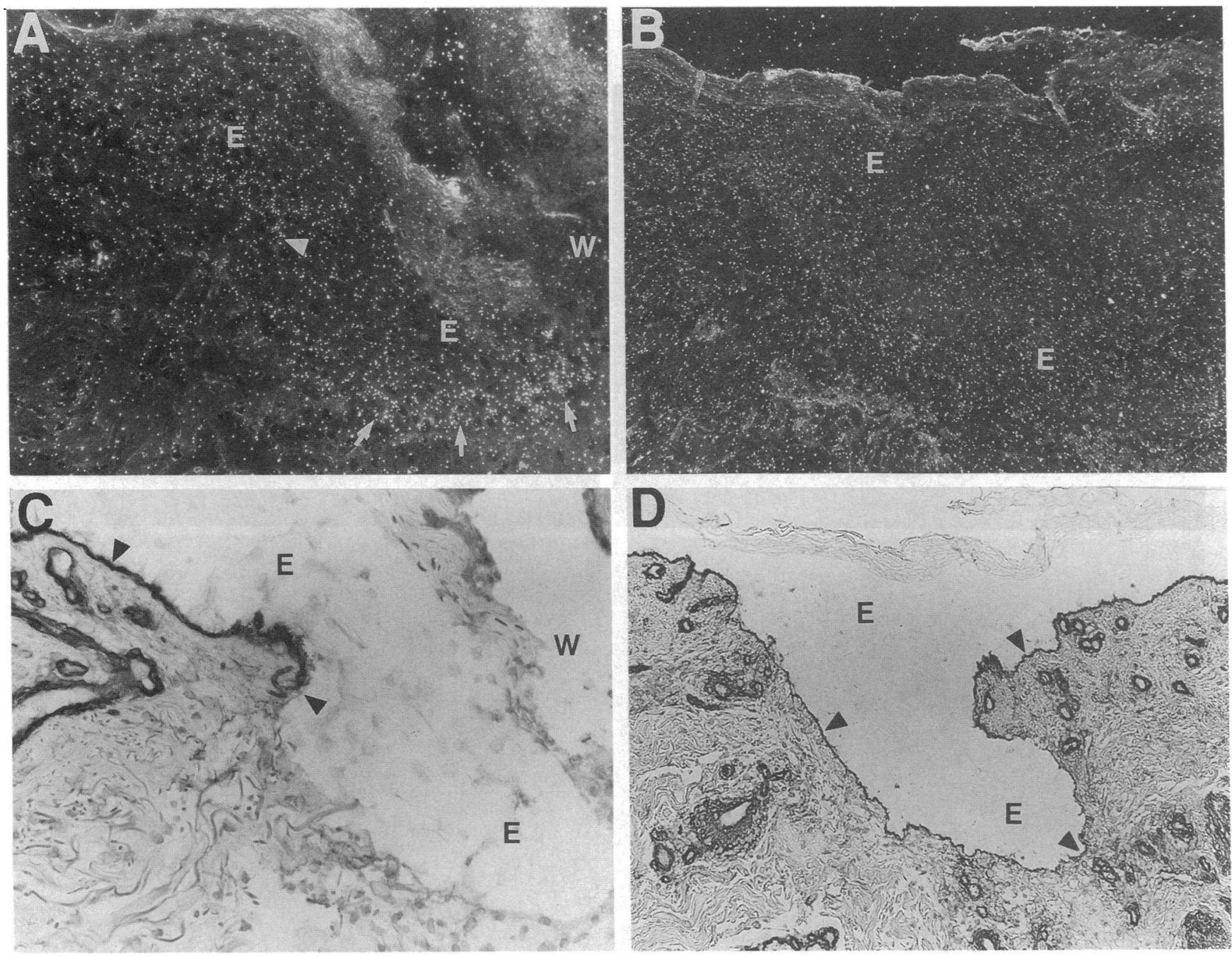

Figure 3. Localization of collagenase mRNA and type IV collagen immunostaining in acute wounds. Parallel sections were hybridized with ${ }^{35}$ S-labeled antisense RNA for interstitial collagenase mRNA ( $A$ and $B$ ) or stained with monoclonal type IV collagen antibody $(C$ and $D$ ). $(A)$ As in chronic wounds, low power dark-field photomicrograph of a section from biopsy taken $3 \mathrm{~d}$ after wounding revealed signal for collagenase mRNA (small arrows) in basal keratinocytes at the healing edge of the wound. The epidermis $(E)$ is seen migrating below the incision wound $(W)$. The arrowhead indicates the approximate extent of the basement membrane. $(C)$ Immunostaining of a parallel section indicates that collagenase-positive cells were not in contact with an intact basement membrane (arrowheads). $(B)$ By $7 \mathrm{~d}$ after wounding, re-epithelialization was completed, and as shown by type IV collagen immunostaining in panel $D$, the basement membrane is intact and continuous. In the dark-field photomicrograph from a parallel section $(B)$, no signal for collagenase mRNA was observed. Autoradiographic exposure for $A$ and $B$ was for 10 d. Magnification: $(A$ and $C) \times 150 ;(B$ and $D) \times 60$. 
hybridization. As we reported $(7,20)$, TIMP-1 mRNA was characteristically seen in macrophage-like cells within inflammatory infiltrates and in perivascular cells. Signal for TIMP-1 mRNA, however, was expressed by one or two epidermal cells in 4/14 samples, but the intensity of the signal for TIMP-1 mRNA in keratinocytes was substantially weaker than the signal found in stromal cells.

Our in vivo observations suggest that contact with the dermal extracellular matrix influences collagenase production by basal keratinocytes. If so, then collagenase would not be expressed in epidermal wounds where the basement membrane remains intact. In the autoimmune disease bullous pemphigoid, epidermal blisters form at the lamina lucida, leaving a mostly intact basement membrane attached to the dermis, yet the area is repaired by re-epithelialization similar to that seen in wound healing. As shown by in situ hybridization, no collagenase mRNA was detected in $3 / 3$ pemphigoid samples (Fig. 4). Similarly, no collagenase mRNA was detected in keratinocytes in pemphigus vulgarus blisters, which occur suprabasilarly and are repaired by re-epithelialization (data not shown).

To assess further the role of cell-matrix interactions and the expression of collagenase, we cultured primary keratinocytes in high $\mathrm{Ca}^{+2}$-containing medium, which promotes differentiation and stratification, on various concentrations of native type I collagen or basement membrane proteins (Matrigel). Collagenase expression was markedly induced in cells plated on collagen, and enzyme production, as quantified by ELISA, correlated directly with the concentration of the collagen substratum (Fig. 5). However, collagenase production was not induced in cells grown on Matrigel and only background levels of enzyme were spontaneously secreted (Fig. 5).

\section{Discussion}

After injury to the skin, activated keratinocytes migrate over the wound bed in direct contact with elements of the provisional and dermal matrices: The findings presented here suggest that these new cell-matrix interactions influence collagenase production in the activated keratinocyte. Indeed, we demonstrated in both chronic and acute wounds that collagenase-expressing keratinocytes were not in contact with an intact basement membrane as assessed by the absence of immu-
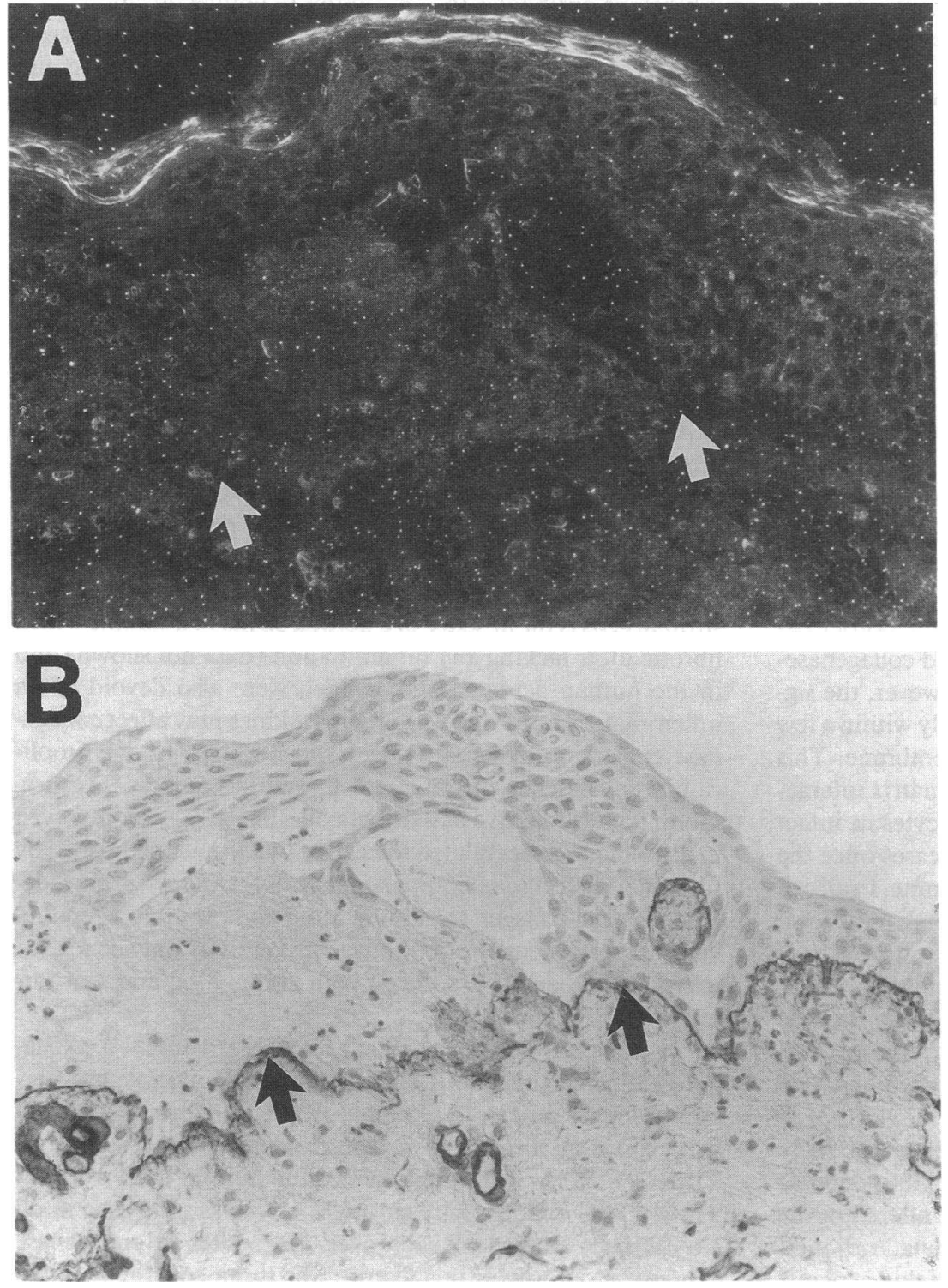

Figure 4. Collagenase is not expressed by keratinocytes in bullous pemphigoid. $(A)$ Dark-field micrograph of a bullous pemphigoid section hybridized for collagenase mRNA. No signal is seen in the injured epidermis or elsewhere. ( $B$ ) Immunostaining of a parallel section for type IV collagen reveals an intact basement membrane (arrows). Autoradiographic exposure was for $10 \mathrm{~d}$. $\times 150$. 


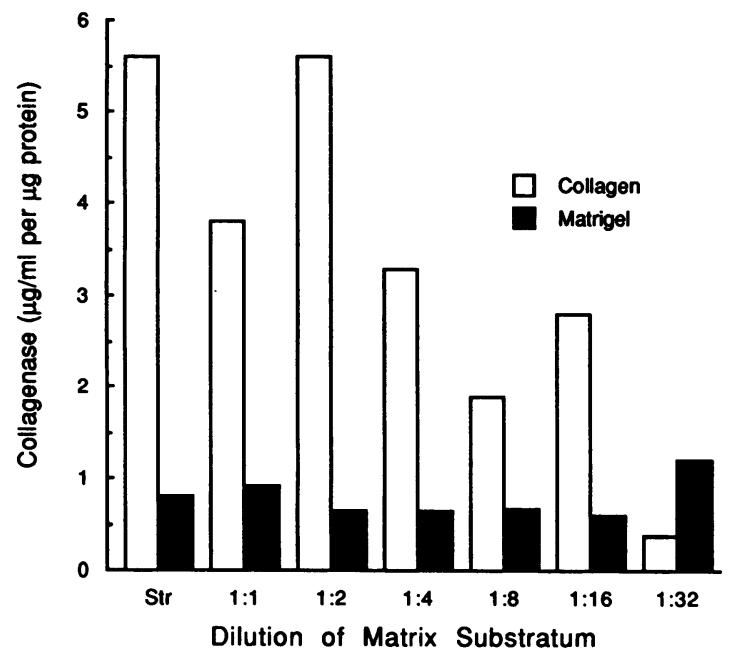

Figure 5. Type I collagen substratum induces collagenase expression in isolated keratinocytes. Normal human keratinocytes were isolated and cultured on dishes coated with either fibrillar type I collagen or basement membrane proteins (Matrigel) as described under Methods. Collagen was added at $750 \mu \mathrm{g} / \mathrm{ml}$ ( Str, straight) and diluted serially to $12 \mu \mathrm{g} / \mathrm{ml}$. Matrigel was added in concentrations ranging from $3,000 \mu \mathrm{g} / \mathrm{ml}$ (Str) to $47 \mu \mathrm{g} / \mathrm{ml}$. After the cells reached confluence, the medium was changed and collected $72 \mathrm{~h}$ later. The levels of accumulated interstitial collagenase were measured by a competitive ELISA.

noreactivity for type IV collagen and laminin. Thus, the homeostatic phenotype of the basal keratinocyte may be maintained by interactions with the underlying basement membrane. Reflecting these in vivo relationships of enzyme expression and underlying matrix, cultured keratinocytes grown on basement membrane proteins (Matrigel) did not produce significant levels of collagenase, whereas cells grown on type I collagen expressed markedly increased levels. Consistent with our data, Petersen et al. ( 31 ) have demonstrated that keratinocytes recognize and migrate on type I collagen substratum and that this interaction results in enhanced collagenase synthesis.

In most of the chronic wounds we studied, the border between immunoreactive basement membrane and collagenasepositive basal keratinocytes was not precise; however, the signal for collagenase mRNA decreased progressively within a few cells overlying the newly formed basement membrane. This observation is consistent with our idea that cell-matrix interactions maintain the phenotype of basal keratinocytes in intact skin and indicates that collagenase expression ceases once the cell establishes contact with the basement membrane. In all cell models studies, collagenase production is regulated at the level of transcription (32-35), and recently we have shown that collagenase mRNA has a half-life of $\sim 6 \mathrm{~h}(32)$. Thus, the weaker signal for collagenase mRNA in basal keratinocytes within the basement membrane border may represent the transcripts that remain and are being degraded after gene expression has been shut off.

We also showed that $\alpha_{5} \beta_{1}$ integrin was present in the same keratinocytes that express interstitial collagenase, thus demonstrating further that cell-matrix interactions are altered in the activated keratinocytes. In agreement with our data, $\alpha_{5} \beta_{1}$ integrin is expressed in keratinocytes migrating out of skin explants
(36), and blocking this integrin receptor has been associated with inhibition of keratinocyte migration on fibronectin matrices (37). Furthermore, Werb et al. (38) found that collagenase expression depends on the capacity to cross-link and cluster $\alpha_{5} \beta_{1}$-receptors on the cell surface of synovial fibroblasts. Although the stimulus that induces integrin expression by keratinocytes is not known, transforming growth factor $\beta 1$, which would be released from degranulated platelets at the initiation of wound repair and from migratory and resident cells during other stages of healing, does upregulate production of $\alpha_{5} \beta 1$ (36). Since the $\alpha_{5} \beta_{1}$ integrin recognizes fibronectin, which is present in the both the provisional and dermal matrices but absent from the epidermal basement membrane, it is doubtful that this receptor transduced induction of collagenase expression by keratinocytes cultured on collagen. Most likely other integrins, such as the type I collagen-binding $\alpha_{2} \beta_{1}$ receptor, which is constitutively expressed on keratinocytes (29), participates in mediating induction of collagenase gene expression. Since keratinocytes are not normally in contact with type I collagen, it is tempting to speculate that basal production of $\alpha_{2} \beta_{1}$ integrin keeps keratinocytes primed and ready to respond to wounding. This will be addressed in future studies by testing if blocking antibodies to $\alpha_{2} \beta_{1}$ inhibit re-epithelialization.

In many specimens of chronic wounds, we detected some expression of collagenase mRNA in stromal cells in the vicinity of the ulcer. Typically, however, only a few scattered positive cells were seen, and the signal per cell was much less than that detected in keratinocytes. Thus, collagenase expression in wound healing is primarily a response of the migrating epidermis. In contrast to the idea that cell-matrix interactions are required for collagenase induction in keratinocytes, enzyme expression in stromal cells may be regulated by cytokines released by advancing granulation tissue (39). Upregulation of collagenase gene expression by fibroblasts is mediated by various inflammatory agents including IL-1, TNF- $\alpha$, and PDGF (40, 41), and macrophage stimulation of collagenase biosynthesis is induced by bacterial endotoxin (19). In human fibroblasts, epidermal growth factor also induces expression of collagenase (40), and this cytokine may be released from platelets during wound healing (42). Supportive of the requirement of inflammatory mediators for expression of collagenase by stromal cells is the absence of a dermal signal in a sample with a fibrotic ulcer lacking any inflammation (data not shown) and in the human acute wounds, which were also devoid of an inflammatory infiltrate. Although cytokines may affect collagenase expression by keratinocytes, our findings strongly implicate a dependence on precise cell-matrix contacts. Furthermore, the degradative activity of collagenase may be involved in distinct processes related to healing that are accomplished by the different cellular compartments. Keratinocytes may degrade dermal collagen to aid migration and promote re-epithelialization, whereas stromal collagenase activity may be related to tissue remodeling associated with granulation and scar formation.

In three samples, weak signal for TIMP-1 mRNA colocalized with collagenase mRNA in migrating keratinocytes. Keratinocytes are capable of secreting TIMP-1 in vitro (43) and, in fact, Jancic et al. (44) have detected temporal expression of TIMP-1 mRNA at the edges of healing human burn wounds. TIMP-1 was expressed by stromal or perivascular cells in all our samples studied, usually away from sites of collagenase expression. However, this feature was more consistent in the 
pyogenic granulomas than in the nonspecific ulcers, the former of which are characterized by extensive, proliferating blood vessels and where TIMP-1 mRNA was frequently observed in a perivascular distribution. This suggests that keratinocyte-derived collagenase is allowed to act without impedance from TIMP-1.

In culture, human keratinocytes on plastic (45) or on collagen produce $92-\mathrm{kD}$ gelatinase $(46,47)$, but in vivo we detected no expression of this enzyme in normal or wounded skin epithelium. These apparently discrepant results may be explained by different factors. Potentially, cell culture may artificially induce gelatinase expression. In fact, we have only detected expression of $92-\mathrm{kD}$ gelatinase in skin specimens with eosinophils and occasional macrophages $(20,21,25)$. In addition, upregulation of $92-\mathrm{kD}$ gelatinase is seen in the epithelial layer of healing rabbit corneal wounds (48), and hence, the healing response in the cornea may be distinct from that in the skin. Since neutrophils store $92-\mathrm{kD}$ gelatinase but do not actively make it (25), this enzyme may be involved in a wound healing response when these cells are present. Finally, Salo et al. (45) have suggested that gelatinases may be important in releasing keratinocytes from the basement membrane before lateral movement at the beginning of epithelial wound healing. Since we studied mostly chronic ulcers, metallogelatinases may have been expressed at earlier stages. Matrilysin may be relevant in wound healing by participating in the activation of plasminogen activator (49) as keratinocytes dissect their way through the provisional matrix in granulation tissue. However, this enzyme was not detected in keratinocytes or inflammatory cells in this study.

In summary, our studies show that production of interstitial collagenase mRNA by basal keratinocytes associated with re-epithelialization is a general phenomenon of wound healing in skin. In addition, our studies demonstrate that collagenase is the predominant metalloproteinase expressed in healing wounds. Collagenolytic activity may contribute to removal of damaged tissue or facilitate the migration of keratinocytes through dermal connective tissue. Based on our findings that the collagenase-positive basal cells are not in contact with an intact basement membrane and that type I collagen stimulates keratinocyte collagenase production in vitro, we hypothesize that migrating keratinocytes actively involved in re-epithelialization acquire a collagenolytic phenotype upon contact with the dermal matrix.

\section{Acknowledgments}

We thank Dr. Gregory I. Goldberg, Washington University, for the collagenase and 72- and 92-kD gelatinase cDNAs, Dr. David Carmichael, Synergen Corp., for the human TIMP cDNA, Dr. John A. McDonald, for antibodies, and Dr. Lynn Matrisian, Vanderbilt University, for the human matrilysin cDNA. We also express our appreciation to Drs. Edmond C. Crouch and Elise Chang for their pathology expertise, and Jill Roby and Teresa Tolley for excellent technical assistance.

This work was supported by grants HL29594 and AR35805 and Training Grant AR07824 from the National Institutes of Health. U.K. Saarialho-Kere is a recipient of a Dermatology Foundation Fellowship Award sponsored by Avon Products, Inc., and acknowledges partial support from The Finnish Cultural Foundation.

\section{References}

1. Welgus, H. G., J. J. Jeffrey, and A. Z. Eisen. 1981. The collagen substrate specificity of human skin fibroblast collagenase. J. Biol. Chem. 256:9511-9515.
2. Welgus, H. G., C. J. Fliszar, J. L. Seltzer, T. M. Schmid, and J. J. Jeffrey. 1990. Differential susceptibility of type $X$ collagen to cleavage by two mammalian collagenases and $72 \mathrm{kD}$ type IV collagenase. J. Biol. Chem. 265:1352113527.

3. Matrisian, L. M. 1992. The matrix-degrading metalloproteinases. BioEssays. 14:455-463.

4. Hibbs, M. S., K. A. Hasty, J. M. Seyer, A. M. Kang, and C. L. Mainardi. 1985. Biochemical and immunological characterization of secreted forms of human neutrophil gelatinase. J. Biol. Chem. 260:2493-2500.

5. Murphy, G., M. I. Cockett, R. V. Ward, and A. J. P. Docherty. 1991. Matrix metalloproteinase degradation of elastin, type IV collagen and proteoglycan. A quantitative comparison of the activities of $95 \mathrm{kDa}$ and $75 \mathrm{kDa}$ gelatinases, stromelysins-1 and -2 and punctuated metalloproteinase (PUMP). Biochem. J. 277:277-279.

6. Matrisian, L. M. 1990 . Metalloproteinases and their inhibitors in matrix remodeling. Trends Genet. 6:121-125.

7. Saarialho-Kere, U. K., E. S. Chang, H. G. Welgus, and W. C. Parks. 1992. Distinct localization of collagenase and TIMP expression in wound healing associated with ulcerative pyogenic granuloma. J. Clin. Invest. 90:1952-1957.

8. Stenn, K. S., and R. Malhotra. 1992. Epithelialization. In Wound Healing: Biochemical and Clinical Aspects. I. K. Cohen, R. F. Diegelmann, and W. J. Linblad, editors. W. B. Saunders Company, Philadelphia. 115-127.

9. Marinkovich, M. P., G. P. Lunstrum, D. R. Keene, and R. E. Burgeson. 1992. The dermal-epidermal junction of human skin contains a novel laminin variant. J. Cell Biol. 119:695-703.

10. Clark, R. A. F., J. M. Lanigan, P. DellaPelle, E. Manseau, H. F. Dvorak, and $R$. B. Colvin. 1982. Fibronectin and fibrin provide a provisional matrix for epidermal cell migration during wound re-epithelialization. J. Invest. Dermatol. 79:264-269.

11. Woodley, D. T., P. M. Bachmann, and E. J. O'Keefe. 1988. Laminin inhibits human keratinocyte migration. J. Cell. Physiol. 136:140-146.

12. Hertle, M. K., J. C. Adams, and F. M. Watt. 1991. Integrin expression during human epidermal development in vivo and in vitro. Development (Camb.). 112:193-206.

13. Olerud, J. E., A. M. Gown, J. Bickembach, B. Dale, and G. F. Odland. 1988. An assessment of human epidermal repair in elderly normal subjects using immunohistochemical methods. J. Invest. Dermatol. 90:845-850.

14. Prosser, I. W., K. R. Stenmark, M. Suthar, E. C. Crouch, R. P. Mecham, and W. C. Parks. 1989. Regional heterogeneity of elastin and collagen gene expression in intralobar arteries in response to hypoxic pulmonary hypertension as demonstrated by in situ hybridization. Am. J. Pathol. 135:1073-1088.

15. Goldberg, G. I., S. M. Wilhelm, A. Kronberger, E. A. Bauer, G. A. Grant, and A. Z. Eisen. 1986. Human fibroblast collagenase. Complete primary structure and homology to an oncogene transformation-induced rat protein. J. Biol. Chem. 261:6600-6605.

16. Wilhelm, S. M., I. E. Collier, B. L. Marmer, A. Z. Eisen, G. A. Grant, and G. I. Goldberg. 1989. SV40-transformed human lung fibroblasts secrete a 92-kDa type IV collagenase which is identical to that secreted by normal human macrophages. J. Biol. Chem. 264:17213-17221.

17. Carmichael, D. F., A. Sommer, R. C. Thompson, D. C. Anderson, C. G. Smith, H. G. Welgus, and G. P. Stricklin. 1986. Primary structure and cDNA cloning of human fibroblast collagenase inhibitor. Proc. Natl. Acad. Sci. USA. $83: 2407-2411$.

18. Collier, I. E., S. M. Wilhelm, A. Z. Eisen, B. L. Marmer, G. A. Grant, J. L. Seltzer, A. Kronberger, C. S. He, E. A. Bauer, and G. I. Goldberg. 1988. H-ras oncogene-transformed human bronchial epithelial cells (TBE-1) secrete a single metalloprotease capable of degrading basement membrane collagen. J. Biol. Chem. 263:6579-6587.

19. Busiek, D. F., F. P. Ross, S. McDonnell, G. Murphy, L. M. Matrisian, and H. G. Welgus. 1992. The matrix metalloprotease matrilysin (PUMP) is expressed in developing human mononuclear phagocytes. J. Biol. Chem. 13:9087-9092

20. Saarialho-Kere, U. K., E. S. Chang, H. G. Welgus, and W. C. Parks. 1993. Expression of interstitial collagenase, $92 \mathrm{kDa}$ gelatinase, and TIMP-1 in granuloma annulare and necrobiosis lipoidica diabeticorum. J. Invest. Dermatol. 100:335-342.

21. Ståhle-Bäckdahl, M., B. D. Sudbeck, A. Z. Eisen, H. G. Welgus, and W. C. Parks. 1992. Expression of $92 \mathrm{kDa}$ type IV collagenase by eosinophils associated with basal cell carcinoma. J. Invest. Dermatol. 99:497-503.

22. Sudbeck, B. D., J. J. Jeffrey, H. G. Welgus, R. P. Mecham, D. McCourt, and W. C. Parks. 1992. Purification and characterization of bovine collagenase and tissue inhibitor of metalloproteinases. Arch. Biochem. Biophys. 293:370376.

23. Roman, J., and J. A. McDonald. 1992. Expression of fibronectin, the integrin $\alpha 5$, and smooth muscle $\alpha$-actin in heart and lung development. Am.J. Respir. Cell Mol. Biol. 6:474-480.

24. Harlow, E., and D. Lane. 1988. Antibodies: A Laboratory Manual. Cold Spring Harbor Laboratory, Cold Spring Harbor, NY. 313-315.

25. Ståhle-Bäckdahl, M., and W. C. Parks. 1993. 92 kDa gelatinase is actively expressed by eosinophils and secreted by neutrophils in invasive squamous cell carcinoma. Am. J. Pathol. 142:995-1000. 
26. Senior, R. M., G. L. Griffin, C. J. Fliszar, S. D. Shapiro, G. I. Goldberg and H. G. Welgus. 1991. Human 92-kilodalton and 72-kilodalton type IV collagenases are elastases. J. Biol. Chem. 266:7870-7875.

27. Pentland, A. P., S. C. Jacobs, M. Mahoney, and M. J. Holzman. 1990 Ultraviolet light potentiates histamine-induced release of prostaglandin in cultured human keratinocytes: a mechanism for irradiation erythema. J. Clin. Invest. 86:566-574.

28. Cooper, T. W., E. A. Bauer, and A. Z. Eisen. 1982. Enzyme-linked immunosorbent assay for human skin collagenase. Collagen Rel. Res. 3:205-211.

29. Pellegrini, G., M. De Luca, G. Orecchia, F. Balzac, O. Cremona, P. Savoia, R. Cancedda, and P. C. Marchisio. 1992. Expression, topography, and function of integrin receptors are severely altered in keratinocytes from involved and uninvolved psoriatic skin. J. Clin. Invest. 89:1783-1795.

30. Docherty, A. J. P., J. O. O'Connell, T. Crabbe, S. Angal, and G. Murphy. 1992. The matrix metalloproteinases and their natural inhibitors: prospects for treating degenerative tissue diseases. Trends Biotechnol. 10:200-207.

31. Petersen, M. J., D. T. Woodley, G. P. Stricklin, and E. J. O'Keefe. 1992 Synthesis and regulation of keratinocyte collagenase. In Matrix Metalloprotein ases and Inhibitors. H. Birkedahl-Hansen, Z. Werb, H. Welgus, and H. V. Wart, editors. Gustav Fisher, Stuttgart. 192-197.

32. Saarialho-Kere, U. K., H. G. Welgus, and W. C. Parks. 1993. Divergent mechanisms regulate interstitial collagenase and $92 \mathrm{kDa}$ gelatinase expression in human monocytic-like cells exposed to bacterial endotoxin. J. Biol. Chem. 268:17354-17361.

33. Shapiro, S., G. A. D. Doyle, W. C. Parks, T. J. Ley, and H. G. Welgus. 1993. Divergent mechanisms regulate the production of collagenase and TIMP during monocytic differentiation. Biochemistry. 32:4286-4292.

34. Angel, P., and M. I. Karin. 1992. Specific members of the jun protein family regulate collagenase expression in response to various extracellular stim uli. In Matrix Metalloproteinases and Inhibitors. H. Birkedahl-Hansen, Z. Werb H. Welgus, and H. V. Wart, editors. Gustav Fisher, Stuttgart. 156-164

35. Auble, D. T., and C. E. Brinkerhoff. 1991. The AP-1 sequence is necessary but not sufficient for phorbol induction of collagenase in fibroblasts. Biochemis try. 30:4629-4635.

36. Guo, M., L. T. Kim, S. K. Akiyama, H. R. Gralnick, K. M. Yamada, and F. Grinnell. 1991. Altered processing of integrin receptors during keratinocyte activation. Exp. Cell. Res. 195:315-322.

37. Kim, J. P., K. Zhang, R. H. Kramer, T. J. Schall, and D. T. Woodley. 1992. Integrin receptors and RGD sequences in human keratinocyte migration unique anti-migratory function of $\alpha 3 \beta 1$ epiligrin receptor. J. Invest. Dermatol. 5:764-770.
38. Werb, Z., P. M. Tremble, O. Behrendtsen, E. Crowley, and C. H. Damsky. 1989. Signal transduction through the fibronectin receptor induces collagenase and stromelysin gene expression. J. Cell. Biol. 109:877-889.

39. Porras-Reyez, B. H., H. C. Blair, J. J. Jeffrey, and T. A. Mustoe. 1991 Collagenase production at the border of granulation tissue in a healing wound: macrophage and mesenchymal collagenase production in vivo. Connect. Tissue Res. 27:63-71.

40. Edwards, D. R., G. Murphy, J. J. Reynolds, S. E. Whitham, A. J. P. Docherty, P. Angel, and J. K. Heath. 1987. Transforming growth factor modulates the expression of collagenase and metalloproteinase inhibitor. EMBO (Eur. Mol. Biol. Organ.) J. 6:1899-1904.

41. Unemori, E. N., M. S. Hibbs, and E. P. Amento. 1991. Constitutive expression of $92-\mathrm{kD}$ gelatinase (type IV collagenase) by rheumatoid synovial fibroblasts and its induction in normal human fibroblasts by inflammatory cytokines. J. Clin. Invest. 88:1656-1662.

42. McKay, I. A., and I. M. Leigh. 1991. Epidermal cytokines and their roles in cutaneous wound healing. Br. J. Dermatol. 124:513-518.

43. Welgus, H. G., and G. P. Stricklin. 1983. Human skin fibroblast collagenase inhibitor: comparative studies in human connective tissues, serum and amniotic fluid. J. Biol. Chem. 258:12259-12264.

44. Jancic, V., L. Liying, B. Wenczak, L. B. Nanney, and G. P. Stricklin. 1992. Detection of collagenase and tissue inhibitor of metalloproteinases in healing human burn wounds by in situ hybridization. J. Invest. Dermatol. 98:623 (Abstr.)

45. Salo, T., J. G. Lyons, F. Rahemtulla, H. Birkedal-Hansen, and H. Larjava. 1991. Transforming growth factor- $\beta 1$ up-regulates type IV collagenase expression in cultured human keratinocytes. J. Biol. Chem. 11436-11441.

46. Woodley, D. T., T. Kalebec, A. J. Baines, W. Link, M. Prunieras, and L. Liotta. 1986. Adult human keratinocytes migrating over nonviable dermal collagen produce collagenolytic enzymes that degrade type I and type IV collagen. $J$. Invest. Dermatol. 4:418-423.

47. Sarret, Y., D. T. Woodley, G. I. Goldberg, A. Kroneberger, and K. C. Wynn. 1992. Constitutive synthesis of a 92-kDa keratinocyte-derived type IV collagenase is enhanced by type I collagen and decreased by type IV collagen matrices. J. Invest. Dermatol. 99:836-841.

48. Fini, M. E., M. T. Girard, and M. Matsubara. 1992. Collagenolytic/gelatinolytic enzymes in corneal wound healing. Acta Ophthalmol. 70:26-33.

49. Marcotte, P. A., I. M. Kozan, S. A. Dorwin, and J. M. Ryan. 1992. The matrix metalloproteinase Pump-1 catalyzes formation of low molecular weight (pro)urokinase in cultures of normal human kidney cells. J. Biol. Chem. 267:13803-13806 